Elahe Aleebrahim-Dehkordi ${ }^{1}$, Niloofar Deravi', Ali Reyhanian ${ }^{3}$,

Shirin Saberianpour ${ }^{4}$, Melika Mokhtari ${ }^{5}$, Ali Hasanpour-Dehkordi ${ }^{*}$

\title{
CHRONIC NON-COMMUNICABLE DISEASES IN THE EPIDEMIC (COVID-19): INVESTIGATION OF RISK FACTORS, CONTROL AND CARE
}

\author{
${ }^{1}$ Medical Plants Research Center, Basic Health Sciences Institute, \\ Shahrekord University of Medical Sciences, Shahrekord, Iran. \\ ${ }^{2}$ Student Research Committee, School of Medicine, \\ Shahid Beheshti University of Medical Sciences, Tehran, Iran. \\ ${ }^{3}$ Student Research Committee, Shahrekord University of Medical Sciences, Shahrekord, Iran. \\ ${ }^{4}$ Vascular and endovascular surgery research center, Mashhad University of medical Science, Mashhad, Iran. \\ ${ }^{5}$ Student Research Committee, Dental Faculty, Tehran Medical Sciences, \\ Islamic Azad University, Tehran, Iran. \\ ${ }^{6}$ Social Determinants of Health Research Center, School of Allied Medical Sciences, \\ Shahrekord University of Medical Sciences, Shahrekord, Iran.
}

\begin{abstract}
INTRODUCTION. Chronic non-communicable diseases (NCDs), global health problem and it is a threat to health and the development of countries. Currently, the number of people with COVID-19 as well as the resulting death toll is rising sharply worldwide. People with underlying diseases may be at greater risk.

AIM. The purpose of the present study was to investigate the chronic non-communicable diseases in the epidemic (COVID-19): Investigation of risk factors, control and care.

METERIALS AND METHODS. To access the articles, including international databases Scopus, PubMed, Web of Science, Embase were searched using the keywords of chronic non-communicable diseases (NCDs), chronic, risk factors, prevention and control, self-efficacy and self-care and their various combinations using AND/OR operators. No language restrictions were applied to the search process.

RESULTS. Based on the evidence, NCDs, exacerbate the negative consequences of COVID-19. according to the results of this study, Among the patients admitted with COVID-19, The most common underlying diseases, were in these people, include, cardiovascular disease, hypertension, chronic obstructive pulmonary disease (COPD), smoking, malignancy, chronic kidney disease, and diabetes mellitus. Obesity may be considered as a potential COVID-19 risk factor.

CONCLUSIONS. Although lifestyle, nutrition, and medical interventions are important for the early prevention of NCDs, having the tools and resources to use information more effectively is more important One of the determining and effective factors in maintaining health and preventing the aggravation of signs and symptoms of the disease COVID-19, especially in chronic diseases, is to perform self-care behaviors.
\end{abstract}

Keywords: NCDs, COVID-19, SARS-CoV-2, risk factors, control measures, lifestyle, self-care 


\section{INTRODUCTION}

Chronic non-communicable diseases (NCDs), global health problems and it is a threat to the health and the development of countries $(1,2)$. NCDs, cover a wide range of diseases. The incidence of these diseases, is associated, with some behavioral factors, including, Tobacco smoking, inadequate physical activity, excessive alcohol consumption, and unhealthy diet $(3,4)$. Among NCDs, cardiovascular diseases, hypertension, diabetes, various cancers, stroke, chronic respiratory diseases and obesity are the most common causes of death $(5,6)$. Since, most of these diseases, have no definitive treatment, and requires long-term care and control of the disease, and its complications. Therefore, effective intervention strategies, especially in the field of primary prevention and lifestyle modification, is one of the most costeffective measures possible So to prevent NCDs, Identifying risk factors, and preventing and controlling these factors is essential $(7,8)$. In addition to the existing challenges to reduce NCDs, the high costs of the disease, but a lot of on health systems world pressure. Report of several people, suffering from unusual pneumonia, at the beginning of the New Year 2020, to the (WHO) from China, Introduced a new type of coronavirus as a cause of a new respiratory disease. With the rapid spread of the disease in China and then to other parts of the world, Disease COVID-19 caused great concern and panic among the people of the world. The outbreak of the virus was a cause of public health emergencies around the world $(9,10)$.The virus mainly affects the respiratory system of people with the disease and is spreading (11). Currently, the number of people with COVID-19 as well as the resulting death toll is rising sharply worldwide (12). However, most of those who died, had a history of underlying diseases, such as, high blood pressure, diabetes, or cardiovascular disease, in which there, immune systems were weakened (13). Given the importance of NCDs, and the lack of attention to the follow-up and treatment of these diseases, with the COVID-19 epidemic and the fear created by this viral disease worldwide, the aims present study Was to Investigation of risk factors, control and care of chronic non-communicable disease.

\section{METERIALS AND METHODS}

To access the articles, including international databases Scopus, PubMed, Web of Science, Embase were searched using the keywords of chronic noncommunicable diseases, risk factors, prevention and control, self-efficacy and self-care and their various combinations using AND/OR operators. No language restrictions were applied to the search process

\section{CARDIOVASCULAR DISEASES}

Cardiovascular disease, is the most common cause of death in most countries of the world, and the most important cause of disability. Despite rapid diagnostic and therapeutic advances, patients with heart attack, die and those who survive, never fully recover and return to normal life. These diseases impose huge costs on the health care systems of countries (14). It seems, that the changes made, in the lifestyle, For example, high intake of processed foods rich in saturated fats, low levels of physical activity, with an increased prevalence of obesity, and Type 2 diabetes, has led to a progressive increase in the prevalence of risk factors for cardiovascular disease and coronary heart disease (CHD) in developed countries (15). Independent risk factors, for this disease, include Family history of disease, smoking, diabetes, hypertension and hyperlipidemia, sedentary lifestyle, Increasing age, Gender and obesity (16). One of the determining and effective factors in maintaining health and preventing the aggravation of signs and symptoms of the disease, is self-care (17). That reducing the number of hospitalizations mortality and treatment costs (18). Cardiovascular disease, had the highest prevalence among diseases that put patients at higher risk of COVID-19 threats. In these patients, decreasing the pro-inflammatory cytokines, leads to a weaker immune function may account for this condition. COVID-19 binds to ACE2, which is present in lung cells. This allows the virus to attack and cause respiratory illness Also, in this case, similar results have been observed for the MERS-CoV virus $(19,20)$.

\section{HYPERTENSION}

Hypertension affects more than 1.2 billion people globally and is currently the most expensive and critical public health issue (21). Which cause 1 in 9 annual deaths in the United States (22). Hypertension is a multifactorial disorder that includes genetic and environmental factors, along with behaviors that confer risk (20). Studies have shown Hypertension is associated with increased disease progression in patients with COVID-19. According to the findings, hypertension seems to be the strongest predictor of disease severity and death in COVID-19 patients. A clinical trial in Wuhan, China, showed that $23.7 \%$ of patients admitted to the intensive care unit have high blood pressure, and in general, high blood pressure is associated with COVID-19 disease. Attention to the Hypertension disease is important for Covid patients in 
several ways; during the illness, the worsening of the disease and the prognosis of the disease is important (23).

\section{CHRONIC OBSTRUCTIVE PULMONARY DISEASE (COPD)}

COPD is the world's third leading cause of death; and caused 3.2 million deaths in 2017, a toll that is expected to approach 4.4 million by 2040 . COPD affects many people in wealthy, middle-income and low-income countries with a global prevalence of $10.1 \%$ (24). COPD with increased changes in local / systemic inflammatory response, host immunodeficiency, microbiome imbalance, persistent mucosal production, structural damage, and use of inhaled corticosteroids has been increasing risk of COVID-19 disease, worsening and mortality. People with COPD have been reported to be more than five times more likely to have a severe COVID-19 infection. Patients with a history of COPD should take more restrictive measures to minimize the potential for exposure to SARS-CoV-2 and contact with suspected or confirmed COVID-19 cases (25).

\section{CANCER}

Cancer is the second major cause of death worldwide, representing an estimated 9.6 million deaths in 2018. Liver, prostate, Lung, stomach and colorectal cancer are the most frequent forms of cancer in men, while thyroid, colorectal, breast, cervical and lung cancer are the most frequent in women (26). Cancer patients, Due to the condition of the disease, weakening of the immune system and the treatments performed, including chemotherapy and surgery, more than other people, at risk of infection are COVID-19 (27). Among the types of cancers, it seems that people with all types of lung malignancies, are the most susceptible, and should consider all control and health measures (28). Studies have demonstrated an elevated risk of COVID-19 in patients with cancer. Also Patients with thoracic malignancy in the elderly and smokers are more likely to develop COVID-19 (29). However, the results of different studies will raise concerns that the small sample size of different cancer studies could show different biological responses with different therapeutic diversity, different types of cancer, and the size of the selected population. Studies results does not represent the entire population with cancer. On the other hand, long-term therapeutic effects such as suppression of the immune system should not be neglected (30).

\section{SMOKING}

Tobacco use is the single greatest preventable cause of disease and death in the United States and reportedly accounts for one third of all cancer deaths in many Western countries $(31,32)$. The results also show that smokers are more susceptible to viral infections, including coronaviruses. Smokers have been reported to have unregulated ACE2 in remodeled cell types (33). Although smoking can exacerbate COVID-19 due to lung damage, the link between smoking and the disease is still unclear. It is noteworthy, however, that the consequences of COVID-19 are greater in smokers and COPD than in others (34). There is speculation about the link between Acute Respiratory Syndrome (SARS-CoV-2) and smoking. In the meantime, studies were performed on the relation among infection, hospitalization and mortality of people with COVID-19 and a history of smoking. The data show that smoking dramatically increases the expression of the angiotensinchron- 2 transmitter gene, a receptor for the acute respiratory syndrome of acute coronavirus 2 , which may explain the high sensitivity to COVID-19 in smokers (35).

\section{CHRONIC KIDNEY DISEASE (CKD)}

CKD in the United States affects 47 million individuals, and is related to substantial health care costs, mortality, and morbidity. Because this illness can advance silently to advanced stages, early detection is essential for initiating timely interventions (36). CKD is an irreversible and progressive degradation of the function of the renal (37). Although the main involvement in COVID-19 is involvement of the alveoli and the respiratory system, involvement of other organs has also been reported in various individuals. Reports of renal involvement in people with COVID-19 are limited. Evaluation between markers of acute renal malignancy in connection with COVID-19, which reached the point of hospitalization and mortality of these patients (38). On the other hand, the need to identify the predictors of severe COVID-19 infection is essential for identifying risk classification, re-optimizing hospital resources, and guiding public health recommendations and interventions (39).

\section{DIABETES MELLITUS}

Diabetes mellitus is a significant chronic disease that leads to severe microvascular and macro vascular, with an estimated global prevalence of $9.3 \%$ in 2019, which is expected to rise to $10.2 \%$ in 2030 (40). There is a well-known association between DM and elevated susceptibility to infections, especially in the 
elderly (41). In patients with diabetes, plasma levels of some proinflammatory cytokines are higher than in non-diabetic controls. Increased proinflammatory cytokines in patients included IL-8, IL-6, and TNF- $\alpha$ (42). Hyperglycemia causes the accumulation and production of glycosylated compounds (AGEs), that a key role in the progression and exacerbation of diabetes, and insulin resistance results in the synthesis of potent inflammatory cytokines, including IFN- $\gamma$ and a rise in the basal cytokine secretion $(43,44)$. These findings would provide evidence of immune dysregulation in diabetic patients. Studies have shown that the presence of underlying diseases such as diabetes, hypertension and obesity significantly increases the morbidity and mortality of COVID-19. The potential mechanisms of diabetes in predisposing COVID-19 are still unknown. More research about this subject is needed, but recent studies suggest that diabetes plays a role in modulating host immune responses. Identification of clinical and biochemical parameters that assess the severity of COVID-19 in diabetic individuals with COVID-19 indicates a chance of developing the disease and malignancy in diabetic individuals (45).

\section{OTHER CHRONIC NON-COMMUNICABLE DISEASES (NCDS) \\ COVID-19 AND RHEUMATOLOGIC DISEASES}

A variety of inflammatory disorders are observed in rheumatologic diseases from different types of arthritis, and autoimmune disorders of the connective tissue to vasculitis. Rheumatic patients often take various types of immunosuppressive agents and immunomodulatory therapies to control their diseases and continue their daily activities. Also, the immune system impairment may be recognized like rheumatologic diseases. Therefore, inflammatory rheumatologic disease patients are prone to an elevated risk of infections in comparison with the general population $(46,47)$. Moreover, as previously mentioned, some underlying diseases including chronic respiratory disease, cardiovascular disease, hypertension, and diabetes mellitus, are among the risk factors of COVID-19 poor outcomes, which are repeatedly recognized in the patients with rheumatologic diseases $(48,49)$. Furthermore, COVID-19 causes acute lung injury and acute interstitial lung disease $(50,51)$.

\section{COVID-19 AND NEUROLOGICAL DISEASES}

Rapid progression of the COVID-19 pandemic provided an increasing body of evidence considering the robust link between COVID-19 and both preand post- neurological complications (52). Definite
COVID-19 patients may be divided into three categories based on their neurological symptoms: neurological manifestations in patients with underlying diseases (such as dizziness, headache, impaired consciousness, (53). epileptic manifestations, seizures, or ataxia $(54,55)$. and stroke (43); neurological expressions associated with neuro-peripheral origin (such as hyposmia, hypo-ageusia, neuralgia) as well as skeletal muscle damage manifestations $(43,56,57)$. The current literature suggests a variable rate of hyposmia and hypogeusia in patients with COVID-19. The hyposmia and hypogeusia prevalence were reported to be 5.1 and $5.6 \%$ in a study on 214 hospitals admitted COVID-19 patients from Wuhan, respectively (43). According to the findings of a European study on 417 patients with mild to moderate COVID-19, olfactory and gustatory dysfunctions were reported by $85.6 \%$ and $88 \%$ of patients, respectively (58). Moreover, nearly $78 \%$ of patients without rhinorrhea or nasal obstruction experienced hyposmia or anosmia (58). In Korea, the prevalence of ageusia and anosmia were $15.7 \%$ and $15.3 \%$ in the early stages of COVID-19 and among asymptomatic to mild COVID-19 patients, respectively (59). In Italy, chemosensory dysfunction was detected in nearly $19.4 \%$ of COVID-19 patients (60). As the emerging reports suggest anosmia as an early COVID-19 manifestation, dedicated anosmia testing could be helpful in early COVID-19 diagnosis (61). Accordingly, it is vital to incorporate targeted neurological care in these patients subgroup, which warranties additional healthcare workforce, reorganization of services, and continuing management of the chronic neurological cases (62). Furthermore, it has been suggested that in the emergency of the COVID-19 pandemic, neurological patients should be separated from non-neurological patients, due to the higher risk posed to their health (63).

\section{COVID-19 AND OBESITY}

Obese people usually have sedentary lives. Also, they suffer insulin resistance, which affects the immune response against microbial agents via pro-inflammatory cytokine levels modulation and impaired macrophage differentiation, which leads to infectious pathogens invasion $(64,65)$. Constant low adiponectin and high leptin level are observed in patients with obesity. Also, they experience a higher level of pro-inflammatory cytokines, including TNFalpha, MCP-1, and IL-6, which are mainly produced in the adipose tissue contributing to immune response impairment $(66,67)$.These factors may induce immune and inflammatory responses. As a result, obesity may be considered as a potential COVID-19 risk factor. According to a recent meta-analysis, obesity 
can be regarded as a considerable risk factor for ICU admission and especially IMV requirement in patients with COVID-19 (68).Therefore, to prevent unfavorable clinical outcomes, obese patients should be watchfully monitored and may require earlier therapy escalation.

\section{CONCLUSION}

Based on the evidence, NCDs, exacerbate the negative consequences of COVID-19. Although lifestyle, nutrition, and medical interventions are important for the early prevention of NCDs, having the tools and resources to use information more effectively is more important; that this, requires the presence of a healthy and efficient health system. Self-care is one of the most important factors in maintaining good health, especially in chronic diseases. Self-care can also control and reduce the severity of symptoms. With self-care, addition to preventing the aggravation of signs and symptoms, is essential in reducing the number of hospitalizations. according to the results of this study, which is based on the evidence, and articles reviewed, Among the patients admitted with COVID-19, The most common underlying diseases, were in these people, include, cardiovascular disease, COPD, smoking, malignancy, chronic kidney disease, and diabetes mellitus. Obesity may be considered as a potential COVID-19 risk factor. Therefore, to prevent unfavorable clinical outcomes, obese patients should be watchfully monitored and may require earlier therapy escalation.so some underlying diseases such as chronic respiratory disease, cardiovascular disease, hypertension, and diabetes mellitus, are among the risk factors of COVID-19 poor outcomes, which are repeatedly recognized in the patients with rheumatologic diseases. Also The strong association between COVID-19 and pre- and post-neurological complications.

\section{Financial support and sponsorship}

Nil.

\section{Conflicts of interest there are no conflicts of interest.}

\section{REFERENCES}

1. Msyamboza KP, Ngwira B, Dzowela T, Mvula C, Kathyola D, Harries AD, et al. The Burden of Selected Chronic Non-Communicable Diseases and Their Risk Factors in Malawi: Nationwide STEPS Survey. PLoS ONE. 2011; 6 (5):e20316. PMID: 21629735 PMCID: PMC3100352 DOI: 10.1371/journal.pone.0020316.
2. Noble N, Paul C, Turon H, Oldmeadow C. Which modifiable health risk behaviours are related? A systematic review of the clustering of Smoking, Nutrition, Alcohol and Physical activity ('SNAP') health risk factors. Prev Med. 2015; 81:16-41. PMID: 26190368.

3. Ory M.G, Ahn S, Jiang L, Smith M.L, Ritter P.L, Whitelaw N, Lorig K. Successes of a national study of the chronic disease self-management program: meeting the triple aim of health care reform. Medical Care. 2013 Nov; 51(11), 992-998. PMID: 24113813 DOI: 10.1097/MLR.0b013e3182a95dd1.

4. Noble N, Paul C, Turon H, Oldmeadow C. Which modifiable health risk behaviours are related? A systematic review of the clustering of Smoking, Nutrition, Alcohol and Physical activity ('SNAP') health risk factors. Prev Med. 2015; 81:16-41. PMID: 26190368.

5. Alwan A, Maclean DR, Riley LM, d'Espaignet ET, Mathers CD, et al. Monitoring and surveillance of chronic non-communicable diseases: progress and capacity in high-burden countries. Lancet. 2010; 376(9755):1861-8. PMID: 21074258.DOI: 10.1016/ S0140-6736 (10)61853-3.

6. Boutayeb A, Boutayeb S. the burden of noncommunicable diseases in developing countries. Int J Equity Health. 2005; 4 (1):2. PMID: 15651987. PMCID: PMC546417 DOI: 10.1186/1475-9276-4-2

7. Maher D, Harries AD, Zachariah R, Emerson D. A global framework for action to improve the primary care response to chronic noncommunicable diseases: a solution to a neglected problem. BMC Public Health. 2009 Sep 22; 9:355.PMID: 19772598 PMCID: PMC2758871 DOI: 10.1186/1471-2458-9-355.

8. Maher D, Smeeth L, Sekajugo J .Health transition in Africa: practical policy proposals for primary care. Bull World Health Organ; 88: 943-948. 2010 Dec 1; 88 (12):943-8. PMID: 21124720 PMCID: PMC2995191 DOI: 10.2471/BLT.10.077891.

9. Tan W, Zhao X, Ma X, Wang W, Niu P, Xu W, et al. A novel coronavirus genome identified in a cluster of pneumonia cases-Wuhan, China 20192020. China CDC Weekly. 2020; 2 (4):61-2. Doi: 10.46234/ccdew2020.017.

10. Chen N, Zhou M, Dong X, Qu J, Gong F, Han Y, et al. Epidemiological and clinical characteristics of 99 cases of 2019 novel coronavirus pneumonia in Wuhan, China: a descriptive study. The Lancet. 2020; 395 (10223):507-13. DOI:https://doi. org/10.1016/S0140-6736 (20)30211-7.

11. Lai CC, Shih TP, Ko WC, Tang HJ, Hsueh PR. Severe acute respiratory syndrome coronavirus 2 (SARS-CoV-2) and coronavirus disease-2019 (COVID-19): The epidemic and the challenges. Int J Antimicrob Agents. 2020; 55(3):105924. PMID: 
32081636 PMCID: PMC7127800 DOI: 10.1016/j. ijantimicag.2020.105924.

12. Bedford J, Enria D, Giesecke J, Heymann DL. COVID-19: towards controlling of a pandemic. Lancet. 2020 Mar 28; 395(10229):1015-1018.PMID: 32197103 PMCID: PMC7270596 DOI: 10.1016/ S0140-6736 (20)30673-5

13. Sun P, Qie S, Liu Z, et al. Clinical Characteristics of 50466 Patients with 2019-Ncov Infection. Medrxiv. In press 2020.

14. Roger, Veronique. L. et al. «Executive summary: Heart disease and stroke statistics-2012 update: A report from the American Heart Association. » Circulation. 2012; 125 (1):188.PMID: 32232218 PMCID: PMC7096724.

15. Mattei J, Malik V, Wedick NM, et al. A symposium and workshop report from the Global Nutrition and Epidemiologic Transition Initiative: nutrition transition and the global burden of type 2 diabetes. Br J Nutr. 2012; 108 (7):1325-1335. DOI: 10.1017/ S0007114512003200.

16. Ortega, L.M. \& Matterson, B.J.Hypertension in peritoneal dialysis patients: Epidemiology, pathogenesis, and treatment. J Am Soc Hypertens. 2011; 5(3), 128-136. PMID: 21459067 DOI: 10.1016/j.jash.2011.02.004.

17. Sidani S. Self-care in D.M. Nursing- Sensitive Outcomes. Sudbury, Massachusetts: Jones and Bartlett.2003; 23(2): 63-70.

18. Jane Turner and Brian Kelly, Emotional dimensions of chronic disease. West J Med. 2000; 172 (2): 124-128. Doi: 10.1136/ewjm.172.2.124.

19. Zheng Y-Y, Ma Y-T, Zhang J-Y, Xie X. COVID-19 and the cardiovascular system. Nature Reviews Cardiology.2020; 17(5):259-260.PMID: 32139904 PMCID: PMC7095524 DOI: 10.1038/s41569-0200360-5.

20. Ramchand, J. et al. Plasma ACE2 activity predicts mortality in aortic stenosis and is associated with severe myocardial fibrosis. Cardiovasc Imaging. 2020; 13 (3) 655-664. PMID: 31607667 DOI: 10.1016/j.jcmg.2019.09.005.

21. Rossier BC, Bochud M, Devuyst O. The Hypertension Pandemic: An Evolutionary Perspective. Physiology (Bethesda, Md). 2017; 32 (2):112-25. PMID: 28202622 DOI: 10.1152/ physiol.00026.2016.

22. Slivnick J, Lampert BC. Hypertension and Heart Failure. Heart failure clinics. 2019; 15(4):531-41. PMID: 31472888 DOI: 10.1016/j.hfc.2019.06.007.

23. Beevers G, Lip GYH, O'Brien E. The pathophysiology of hypertension. BMJ. 2001; 322(7291):912-6. PMID: 11302910 PMCID: PMC1120075 DOI: 10.1136/bmj.322.7291.912.

24. Sardu, C., et al., Hypertension, thrombosis, kidney failure, and diabetes: Is COVID-19 an endothelial disease? A comprehensive evaluation of clinical and basic evidence. J Clin Med. 2020; 9 (5): 1417. PMID: 32403217 PMCID: PMC7290769 DOI: $10.3390 / \mathrm{jcm} 9051417$.

25. Celli BR, Wedzicha JA. Update on Clinical Aspects of Chronic Obstructive Pulmonary Disease. N Engl J Med. 2019; 381(13):1257-66. PMID: 31553837 DOI: 10.1056/NEJMra1900500.

26. Lippi, G. and B.M. Henry, Chronic obstructive pulmonary disease is associated with severe coronavirus disease 2019 (COVID-19). Respiratory Medicine, 2020; 167: 105941. PMID: 32421537. PMCID: PMC7154502 DOI: 10.1016/j. rmed.2020.105941.

27. Pranata R, Soeroto AY, Ian H, Lim MA, Santoso $P$, Permana H. Effect of chronic obstructive pulmonary disease and smoking on the outcome of COVID-19. Int J Tuberc Lung Dis. 2020; 10. DOI: 10.5588/ijtld.20.0278.

28. Xia Y, Jin R, Zhao J, Li W, Shen H. Risk of COVID-19 for cancer patients. The Lancet Oncology. 2020 Apr; 21(4):e180. PMID: 32142622 PMCID: PMC7130057 DOI: 10.1016/S14702045(20)30150-9.

29. Liang W, Guan W, Chen R, Wang W, Li J, Xu K, et al. Cancer patients in SARS-CoV-2 infection: a nationwide analysis in China. The Lancet Oncology. 2020; 21 (3):335-7. PMID: 32066541 PMCID: PMC7159000 DOI: 10.1016/S14702045(20)30096-6.

30. Whisenant, J.G., et al., TERAVOLT: Thoracic Cancers International COVID-19 Collaboration. 2020 Jun 8; 37 (6):742-745.PMID: 32425702 PMCID: PMC7229923 DOI: 10.1016/j. ccell.2020.05.008.

31. Xia, Y., et al., Risk of COVID-19 for patients with cancer. The Lancet Oncology, 2020. 21(4): p. e180. Lancet Oncol. 2020; 21(4):e180. PMID: 32142622 PMCID: PMC7130057 DOI: 10.1016/S14702045(20)30150-9.

32. Sasco A, Secretan M, Straif K. Tobacco smoking and cancer: a brief review of recent epidemiological evidence. Lung Cancer. 2004; 45 2:S3-9. PMID: 15552776 DOI: 10.1016/j.lungcan.2004.07.998.

33. Control CfD, Prevention. Current cigarette smoking among adults-United States, 2011. MMWR Morbidity and mortality weekly report. 2012; 61(44):889. PMID: 23134971.

34. Seys LJM, Widagdo W, Verhamme FM, Kleinjan A, Janssens W, Joos GF, et al. DPP4, the Middle East Respiratory Syndrome Coronavirus Receptor, is Upregulated in Lungs of Smokers and Chronic Obstructive Pulmonary Disease Patients. Clinical infectious diseases: an official publication of the Infectious Diseases Society of America. 2018; 66 (1):45-53. PMID: 29020176 PMCID: PMC7108100 DOI: $10.1093 / \mathrm{cid} / \mathrm{cix} 741$. 
35. Zhang J-j, Dong X, Cao Y-y, Yuan Y-d, Yang Y-b, Yan Y-q, et al. Clinical characteristics of 140 patients infected with SARS-CoV-2 in Wuhan, China. Allergy. 2020 Jul; 75(7):1730-1741. PMID: 32077115 DOI: 10.1111/all.14238.

36. Grundy E.J, et al., Smoking, SARS-CoV-2 and COVID-19: A review of reviews considering implications for public health policy and practice. Tobacco Induced Diseases, 2020; 18:58. PMID: 32641924 PMCID: PMC7336902 DOI: 10.18332/ tid/124788.

37. Gaitonde DY, Cook DL, Rivera IM. Chronic Kidney Disease: Detection and Evaluation. American family physician. 2017; 96 (12):776-83. PMID: 29431364.

38. Lopez-Novoa JM, Martinez-Salgado C, Rodríguez-Pena AB, Hernández FJL. Common pathophysiological mechanisms of chronic kidney disease: Therapeutic perspectives. Pharmacology \& Therapeutics. 2010; 128 (1):61-81. PMID: 20600306 DOI: 10.1016/j.pharmthera.2010.05.006.

39. Cheng Y, et al. Kidney disease is associated with inhospital death of patients with COVID-19. Kidney international, 2020.PMID: 32247631 PMCID: PMC7110296 DOI: 10.1016/j.kint.2020.03.005.

40. Henry, B.M. and G. Lippi, Chronic kidney disease is associated with severe coronavirus disease 2019 (COVID-19) infection. International urology and nephrology, 2020; 52 (6):1193-1194. PMID: 32222883 PMCID: PMC7103107 DOI: 10.1007/ s11255-020-02451-9.

41. Saeedi P, Petersohn I, Salpea P, Malanda B, Karuranga S, Unwin N, et al. Global and regional diabetes prevalence estimates for 2019 and projections for 2030 and 2045: Results from the International Diabetes Federation Diabetes Atlas. Diabetes research and clinical practice. 2019; 157:107843. PMID: 31518657 DOI: 10.1016/j. diabres.2019.107843.

42. Joshi N, Caputo GM, Weitekamp MR, Karchmer A. Infections in patients with diabetes mellitus. New England Journal of Medicine. 1999; 341(25):1906-12. PMID: 10601511 DOI: 10.1056/ NEJM199912163412507.

43. Geerlings SE, Hoepelman AI. Immune dysfunction in patients with diabetes mellitus (DM). FEMS Immunology \& Medical Microbiology. 1999; 26 (3-4):259-65. PMID: 10575137 DOI: 10.1111/j.1574695X.1999.tb01397.x.

44. Imani F, Horii Y, Suthanthiran M, Skolnik E, Makita Z, Sharma V, et al. Advanced glycosylation endproduct-specific receptors on human and rat T-lymphocytes mediate synthesis of interferon gamma: role in tissue remodeling. The Journal of experimental medicine. 1993; 178(6):2165-72. PMID: 8245789 PMCID: PMC2191269 DOI: 10.1084/jem.178.6.2165.
45. Vlassara H, Brownlee M, Manogue KR, Dinarello CA, Pasagian A. Cachectin/TNF and IL-1 induced by glucose-modified proteins: role in normal tissue remodeling. Science. 1988; 240 (4858):1546-8. PMID: 3259727 DOI: 10.1126/science.3259727.

46. Muniyappa, R. and S. Gubbi, COVID-19 pandemic, coronaviruses, and diabetes mellitus. American Journal of Physiology-Endocrinology and Metabolism, 2020. 318(5): p. E736-E741. PMID: 32228322 PMCID: PMC7191633 (available on 2021-05-01) DOI: 10.1152/ajpendo.00124.2020.

47. Mehta B, Pedro S, Ozen G, Kalil A, Wolfe F, Mikuls $\mathrm{T}$, et al. Serious infection risk in rheumatoid arthritis compared with non-inflammatory rheumatic and musculoskeletal diseases: a US national cohort study. RMD open. 2019; 5(1):e000935. PMID: 31245055 PMCID: PMC6560658 DOI: 10.1136/ rmdopen-2019-000935.

48. Alarcon GS. Infections in systemic connective tissue diseases: systemic lupus erythematosus, scleroderma, and polymyositis/dermatomycosis's. Infect Dis Clin North Am. 2006; 20(4):849-75. PMID: 17118293 DOI: 10.1016/j.idc.2006.09.007.

49. Nurmohamed MT, Heslinga M, Kitas GD. Cardiovascular comorbidity in rheumatic diseases. Nature reviews Rheumatology. 2015; 11(12):693-704. PMID: 26282082 DOI: 10.1038/ nrrheum.2015.112.

50. Burner TW, Rosenthal AK. Diabetes and rheumatic diseases. Current opinion in rheumatology. 2009; 21(1):50-4. PMID: 19077719 DOI: 10.1097/ BOR.0b013e32831bc0c4.

51. Huang C, Wang Y, Li X, Ren L, Zhao J, Hu Y, et al. Clinical features of patients infected with 2019 novel coronavirus in Wuhan, China. The lancet. 2020; 395(10223):497-506.PMID: 31986264 PMCID: PMC7159299 DOI: 10.1016/S01406736(20)30183-5.

52. Ai T, Yang Z, Hou H, Zhan C, Chen C, Lv W, et al. Correlation of chest CT and RT-PCR testing in coronavirus disease 2019 (COVID-19) in China: a report of 1014 cases. Radiology. 2020; 296(2):E32-E40. PMID: 32101510 PMCID: PMC7233399 DOI: 10.1148/radiol.2020200642.

53. Bhaskar S, Bradley S, Israeli-Korn S, Menon B, Chattu VK, Thomas $\mathrm{P}$, et al. Chronic neurology in COVID-19 era: clinical considerations and recommendations from the REPROGRAM Consortium. Frontiers in neurology. 2020; 11:664. PMID: 32695066 PMCID: PMC7339863 DOI: 10.3389/fneur.2020.00664.

54. Mao L, Jin H, Wang M, Hu Y, Chen S, He Q, et al. Neurologic manifestations of hospitalized patients with coronavirus disease 2019 in Wuhan, China. JAMA neurology. 2020; 77(6):683-90. PMID: 32275288 PMCID: PMC7149362 DOI: 10.1001/ jamaneurol.2020.1127. 
55. Hussain S, Chen Y, Yang Y, Xu J, Peng Y, Wu Y, et al. Identification of novel sub genomic RNAs and no canonical transcription initiation signals of severe acute respiratory syndrome coronavirus. Journal of virology. 2005; 79(9):5288-95. DOI: 10.1128/JVI.79.9.5288-5295.

56. Ashour HM, Elkhatib WF, Rahman M, Elshabrawy HA. Insights into the recent 2019 novel coronavirus (SARS-CoV-2) in light of past human coronavirus outbreaks. Pathogens. 2020; 9(3):186. PMID: 32143502 PMCID: PMC7157630 DOI: 10.3390/ pathogens9030186.

57. Zhou P, Yang X-L, Wang X-G, Hu B, Zhang L, Zhang $\mathrm{W}$, et al. Discovery of a novel coronavirus associated with the recent pneumonia outbreak in humans and its potential bat origin. BioRxiv. 2020; 579(7798):270-273. PMID: 32015507 PMCID: PMC7095418 DOI: 10.1038/s41586-020-2012-7.

58. Filatov A, Sharma P, Hindi F, Espinosa PS. Neurological complications of coronavirus disease (COVID-19): encephalopathy. Cureus. 2020; 12(3). PMID: 32328364 PMCID: PMC7170017 DOI: 10.7759/cureus.7352.

59. Lechien JR, Chiesa-Estomba CM, De Siati DR, Horoi M, Le Bon SD, Rodriguez A, et al. Olfactory and gustatory dysfunctions as a clinical presentation of mild-to-moderate forms of the coronavirus disease (COVID-19): a multicenter European study. European Archives of Oto-RhinoLaryngology. 2020:1-11. PMID: 32253535 PMCID: PMC7134551 DOI: 10.1007/s00405-020-05965-1.

60. Lee Y, Min P, Lee S, Kim S-W. Prevalence and duration of acute loss of smell or taste in COVID-19 patients. Journal of Korean medical science. 2020; 35(18). PMID: 32383370 PMCID: PMC7211515 DOI: $10.3346 / j k m s .2020 .35 . e 174$.

61. Vaira LA, Salzano G, Deiana G, De Riu G. Anosmia and ageusia: common findings in COVID-19 patients. The Laryngoscope. 2020; 130(7):1787. PMID: 32237238 PMCID: PMC7228304 DOI: 10.1002/lary.28692.

62. Zubair AS, McAlpine LS, Gardin T, Farhadian S, Kuruvilla DE, Spudich S. Neuropathogenesis and Neurologic Manifestations of the Coronaviruses in the Age of Coronavirus Disease 2019: A Review. JAMA Neurology. 2020; 77(8):1018-1027.PMID: 32469387 DOI: 10.1001/jamaneurol.2020.2065.

63. Montalvan V, Lee J, Bueso T, De Toledo J, Rivas K. Neurological manifestations of COVID-19 and other coronavirus infections: A systematic review. Clin. Neurol. Neurosurg. 2020; 194:105921. PMID: 32422545 PMCID: PMC7227498 DOI: 10.1016/j. clineuro.2020.105921.

64. Needham EJ, Chou SH, Coles AJ, Menon DK. Neurological implications of COVID-19 infections. NEUROCRIT CARE. 2020; 28:1. PMID: 32346843 PMCID: PMC7188454.DOI: 10.1007/s12028-020-00978-4.

65. Reidy PT, Yonemura NM, Madsen JH, McKenzie AI, Mahmassani ZS, Rondina MT, et al. An accumulation of muscle macrophages is accompanied by altered insulin sensitivity after reduced activity and recovery. Acta Physiologica. 2019; 226 (2):e13251. PMID: 30632274 DOI: 10.1111/apha.13251.

66. Zheng Q, Cui G, Chen J, Gao H, Wei Y, Uede $\mathrm{T}$, et al. Regular exercise enhances the immune response against microbial antigens through upregulation of toll-like receptor signaling pathways. Cellular Physiology and Biochemistry. 2015; 37 (2):735-46. https://doi.org/10.1159/000430391.

67. Richard C, Wadowski M, Goruk S, Cameron L, Sharma AM, Field CJ. Individuals with obesity and type 2 diabetes have additional immune dysfunction compared with obese individuals who are metabolically healthy. BMJ Open Diabetes Research and Care. 2017; 5(1): e000379.PMID: 28761653 PMCID: PMC5530252 DOI: 10.1136/ bmjdrc-2016-000379.

68. Ouchi N, Parker JL, Lugus JJ, Walsh K. Adipokines in inflammation and metabolic disease. Nature reviews immunology. 2011; 11(2):85-97. PMID: 21252989 PMCID: PMC3518031 DOI: 10.1038/ nri2921.

69. Foldi M, Farkas N, Kiss S, Zadori N, Vancsa $\mathrm{S}$, Szako L, et al. Obesity is a risk factor for developing critical condition in COVID-19 patients: A systematic review and meta-analysis. Obesity Reviews.2020; 21(10): el3095. https://doi. org/10.1111/obr.13095.

Received: 28.09.2020

Accepted for publication: 23.10.2020

Address for correspondence:

ali20121968@yahoo.com 